\title{
Expression of membrane and nuclear progesterone receptors in two human placental choriocarcinoma cell lines (JEG-3 and BeWo): Effects of syncytialization
}

\author{
ELENA ZACHARIADES ${ }^{1}$, HELEN FOSTER $^{1}$, ANASTASIA GOUMENOU ${ }^{1,2}$, \\ PETER THOMAS $^{3}$, MARIANN RAND-WEAVER ${ }^{1}$ and EMMANOUIL KARTERIS ${ }^{1}$
}

${ }^{1}$ Centre for Cell Chromosome Biology, Biosciences, School of Health Sciences and Social Care, Brunel University, Uxbridge, UB8 3PH, UK; ${ }^{2}$ University of Crete, Division of Medicine, Department of Obstetrics and Gynecology, Heraklion, Crete, Greece; ${ }^{3}$ Marine Science Institute, University of Texas at Austin, Port Aransas, TX 78373, USA

Received December 17, 2010; Accepted January 19, 2011

DOI: $10.3892 / \mathrm{ijmm} .2011 .657$

\begin{abstract}
A vital function of the human placenta is to produce steroid hormones such as progesterone, which are essential for the maintenance of pregnancy and the onset of parturition. Although choriocarcinoma cell lines are valuable placental models for investigations of steroid hormone actions, little is known about the expression of progesterone receptors (PRs) in these cell lines. Therefore, in this study, the expression of membrane and nuclear PRs was investigated in cultures of fusigenic (BeWo) and non-fusigenic (JEG-3) human choriocarcinoma cell lines. In addition, the effects of an inducer of syncytialization (forskolin) on the PR expression in BeWo cells were assessed. Quantitative RT-PCR revealed that in fully syncytialized BeWo cells (treated with $50 \mu \mathrm{M}$ forskolin for $72 \mathrm{~h}$ ) there was a significant down-regulation of $\mathrm{mPR} \alpha$ and up-regulation of $\mathrm{mPR} \beta$ and of the progesterone membrane component-1 (PGRMC1) when compared with non-syncytialized BeWo cells. Expression of all the mPR and PGRMC1 mRNAs was significantly lower in JEG-3 cells compared to non-syncytialized BeWo cells. Interestingly, expression of PR-B was unaltered between the two BeWo states but was significantly higher in JEG-3 cells. Immunofluorescence analysis revealed that mPR proteins are differentially expressed in these choriocarcinoma cell lines as well as in the human placenta. The data demonstrate that human choriocarcinoma cell lines have a complex system of progesterone signalling involving multiple classes of PRs. The finding that syncytialization is accompanied by changes in the expression of these receptors may suggest that this process influences progesterone signalling.
\end{abstract}

Correspondence to: Dr Emmanouil Karteris, Centre for Cell Chromosome Biology, Biosciences, School of Health Sciences and Social Care, Brunel University, Uxbridge, UB8 3PH, UK

E-mail: emmanouil.karteris@brunel.ac.uk

Key words: placenta, syncytialization, progesterone receptor

\section{Introduction}

Progesterone (P4) plays a key role in the reproductive tract for the initiation and continuation of early pregnancy $(1,2)$, and also prevents preterm labour and spontaneous abortion (3). Progesterone-mediated responses are complex because they are mediated by multiple types of progesterone receptors (PRs). In addition to the two well studied variants of the classic PRs, PR-B (116 kDa) and PR-A (94 kDa), new splicing isoforms have also been detected including PR-C (60 kDa), PR-M (38 kDa), as well as PR-S and PR-T variants that contain novel intronic exons (4).

Numerous research groups have shown that progesterone can also act at the cell surface of many target tissues and cell types to initiate rapid responses, via binding to membrane receptors (5). However, the lack of comprehensive information on the identity and molecular structure of membrane steroid receptors has prevented understanding of critical molecular aspects of steroid actions via this alternative mechanism and a more widespread appreciation of its significance (6). Recently, new genes exhibiting characteristics of a membrane PR (mPR) have been discovered. These include the family of the seven transmembrane domain mPRs as well as the progesterone receptor membrane component 1 (PGRMC1) (7). These receptors are structurally unrelated to the nuclear PR and phylogenetically distinct from the superfamily of G-proteincoupled receptors (GPCRs) $(7,8)$. The mPR family includes PAQRV (mPR $\gamma)$, PAQR VI, PAQR VII (mPR $\alpha)$, PAQR VIII $(\mathrm{mPR} \beta)$ and PAQR IX (9) and the recently described PAQR6 (mPRS) and PAQR9 (mPRe) (10).

Another plausible mechanism mediating the non-genomic actions of progesterone is through PGRMC1. This is a small protein $(28 \mathrm{kDa})$ associated with progesterone binding (11) first isolated from a porcine vascular smooth muscle cell cDNA library (12). This protein consists of 194 amino acids (12) and comprises a single transmembrane domain and a cytoplasmic domain containing three Src homology domains that may be involved in the ligand-dependent signal transduction (13).

The ovaries and the fetoplacental unit are the major sources of progesterone (14). During human pregnancy, progesterone 
levels rise until the third trimester and act in a paracrine or autocrine manner. PRs have been identified in the human placenta. For example, the purported cytoplasmic $60 \mathrm{kDa}$ PR isoform (PR-C) predominates in human fetal membranes and placenta at term (15), whereas $\mathrm{mPR} \alpha$ is localised in placental syncytiotrophoblasts (9). PGRMC1 is also expressed in the human placenta primarily in syncytiotrophoblasts, smooth muscle cells of the placental vasculature, and in villous capillaries (16).

Interestingly, there is no data to date about the expression of PRs in human choriocarcinoma cell lines, which are used extensively as placental experimental models. Syncytialization is the process by which in the human placenta the differentiated syncytiotrophoblast layer is formed by the continual fusion and incorporation of new cytotrophoblasts $(17,18)$. Positioned at the surface of the placental villi the syncytiotrophoblast layer holds a crucial role in regulating transplacental movement of amino acids, fatty acids as well as glucose (19). Nutrients as well as waste products in the human placenta are transported across the syncytiotrophoblast layer by the nutrient transporters expressed on these cells $(19,20)$. Variations in the activity and expression of placental nutrient transporters may be responsible for pregnancy complications such as intrauterine growth restriction IUGR (21). Moreover, the syncytiotrophoblast layer also has synthetic properties (22), including production and secretion of key, to the homeostasis of the pregnancy, hormones (17). In this study, we examined the expression of nuclear and membrane PRs including PGRMC1 in two human placental choriocarcinoma cell lines (BeWo and JEG-3). In addition, we investigated the effects of syncytialization on PR expression.

\section{Materials and methods}

Cell culture. BeWo and JEG-3 cell lines were purchased from the European Collection of Cell Cultures (ECACC, Salisbury, UK). The cells were maintained at standard culture conditions of $5 \% \mathrm{CO}_{2}$ in air at $37^{\circ} \mathrm{C}$. BeWo cells were cultured in Ham F12 (Sigma-Aldrich, UK) containing 10\% heat-inactivated fetal bovine serum (FBS), and $0.5 \%$ penicillin-streptomycin; whereas, JEG-3 cells were maintained in MEME (SigmaAldrich) containing $10 \%$ heat-inactivated FBS, and $0.5 \%$ penicillin-streptomycin, $0.5 \%$ L-glutamine, $0.5 \%$ sodium pyruvate and $0.5 \%$ MEM non-essential amino acids.

Placental tissue. Paraffin-embedded labouring placental tissues were obtained from women delivering at term without any complications ( $>37$ weeks of gestation, $n=3$ ). Ethics approval was granted by the Brunel University Ethics authority.

Syncytialization of BeWo cells. Forskolin (Sigma-Aldrich) was used as an inducer of syncytialization in BeWo cytotrophoblast cells. Syncytialization of BeWo cells was assessed with 50 and $100 \mu \mathrm{M}$ forskolin (in DMSO) over a total period of $72 \mathrm{~h}$, renewing forskolin treatment every $24 \mathrm{~h}$.

RNA isolation, cDNA synthesis and RT-PCR. Total ribonucleic acid (RNA) was isolated using an RNA extraction kit (Sigma-Aldrich), according to the manufacturer's instructions.
RNA concentration was determined by spectrophotometric analysis (NanoDrop; Thermo Scientific, UK) and agarose gel electrophoresis. RNA (500 ng) was reverse-transcribed into cDNA using $5 \mathrm{IU} / \mu \mathrm{l}$ Rase $\mathrm{H}$ reverse transcriptase (Invitrogen, UK). PCR amplification was performed using Taq polymerase (Invitrogen) and oligonucleotide primers as previously described (15). Twenty-eight cycles were performed consisting of an initial denaturing step at $94^{\circ} \mathrm{C}$ for $30 \mathrm{sec}$, annealing at $60^{\circ} \mathrm{C}$ for $1 \mathrm{~min}$ and elongation at $72^{\circ} \mathrm{C}$ for $1 \mathrm{~min}$.

Quantitative RT-PCR. Relative expression of the genes of interest was assessed by quantitative PCR (Q-PCR) on an ABI7400 instrument (Applied Biosystems) using SYBR ${ }^{\circledR}$ Green-PCR reaction mixture (Sigma-Aldrich) and the primers described in Table I. As a negative control for all the reactions, distilled water was used in place of the cDNA. RNAs were assayed from two to three independent biological replicates. As a negative control, distilled water was used in place of the cDNA. For the quantitative PCR, the following equations were used: $\Delta \mathrm{Ct}=\mathrm{Ct}_{\text {(gene of interest) }}-\mathrm{Ct}_{\text {(house keeping gene) }} ; \Delta \Delta \mathrm{Ct}=\Delta \mathrm{Ct}_{\text {(sample) }}$ $-\Delta \mathrm{Ct}_{\text {(calibrator) }}$ and relative quantity $(\mathrm{RQ})=2^{-\Delta \Delta \mathrm{Ct}}$. The RQ value was set up as 1 for the untreated (no supplement) BeWo cells.

Immunofluorescence analysis. Syncytialized and nonsyncitialized BeWo as well as JEG-3 cells were fixed in 4\% paraformaldehyde for $10 \mathrm{~min}$ prior to washes in PBS and incubation with $10 \%$ bovine serum albumin (BSA) for $1 \mathrm{~h}$. Cells were incubated for $1 \mathrm{~h}$ with antibodies against mPRs, PR-B (Santa Cruz Biotechnology, USA), and PGRMC1 (provided by Dr Peter Thomas) at a 1:200 dilution in 1\% BSA/PBS. Cells were then washed with PBS prior to an incubation with a TRITC-conjugated secondary antibody (Santa Cruz Biotechnology) for $1 \mathrm{~h}$. Slides were washed with PBS and mounted in Vectashield ${ }^{\circledR}$ Mounting Medium (Vector Laboratories) containing the dye 4,6-diamido-2-phenylindole (DAPI) to counterstain nuclei. Images were captured using a Plan Apo Neofluor 63X NA 1.25 oil objective (Zeiss) on a Zeiss Axiovert 200M microscope and viewed using the AxioVision software. Images were taken at a set exposure time.

Protein extraction from BeWo cells. BeWo cells were cultured to $80 \%$ confluency, in the presence or absence of forskolin (50 or $100 \mu \mathrm{M}$ up to $72 \mathrm{~h}$ ). Cells were then lysed using $200 \mu \mathrm{l}$ $1 \mathrm{X}$ Laemmli buffer (Sigma-Aldrich) and denatured for $5 \mathrm{~min}$ at $100^{\circ} \mathrm{C}$ before they were cooled on ice.

Western blotting. The samples were separated on a $10 \%$ SDS-polyacrylamide gel and the proteins were transferred to a nitrocellulose membrane. The membrane was blocked in TBS containing $5 \%$ dried milk powder (w/v) and $0.1 \%$ Tween-20, for $1 \mathrm{~h}$ at room temperature. After three washes with TBS- $0.1 \%$ Tween-20, the nitrocellulose membranes were incubated with primary antibodies against total PR, recognising both PR-A and PR-B (Santa Cruz Biotechnology). The primary antiserum was used at a 1:250 dilution overnight at $4^{\circ} \mathrm{C}$. The membranes were washed thoroughly for $30 \mathrm{~min}$ with TBS-0.1\% Tween, before incubation with the secondary HRP-conjugated immunoglobulin $(1: 2,000)$ for $1 \mathrm{~h}$ at room temperature and further washing for $30 \mathrm{~min}$ with TBS-0.1\% Tween-20. Antibody complexes were visualised as previously described (23). 
Table I. Set of primers used for RT-PCR analyses.

\begin{tabular}{|c|c|c|}
\hline Gene & Sequence & PCR product size (bp) \\
\hline $\mathrm{mPR} \alpha$ & $\begin{array}{l}\text { 5'-GCTGTTCACTCACATCCC-3' } \\
\text { 5'-TGGTGCAACCCCCAGA-3' }\end{array}$ & 289 \\
\hline $\operatorname{mPR} \beta$ & $\begin{array}{l}\text { 5'-GCGGCCCTGGTACTGCTGC-3' } \\
\text { 5'-CACGGCCACCCCCACA-3' }\end{array}$ & 200 \\
\hline $\mathrm{mPR} \gamma$ & $\begin{array}{l}\text { 5'-CAGCTGTTTCACGTGTGTGTGATCCTG-3' } \\
\text { 5'-GCACAGAAGTATGGCTCCAGCTATCTGAG-3' }\end{array}$ & 120 \\
\hline $\mathrm{mPR} \delta$ & $\begin{array}{l}\text { 5'-CCCCAACTTCTTCAAGTCCA-3' } \\
\text { 5'-CTGGAAGGAGCTGAGGACAC-3' }\end{array}$ & 114 \\
\hline PGRMC1 & $\begin{array}{l}\text { 5'-TCTGGACTGCACTGTTGTCCTTG-3' } \\
\text { 5'-GCAAACACCTGTTCCTATTCTG-3' }\end{array}$ & 290 \\
\hline$\beta$-actin & $\begin{array}{l}\text { 5'-AAGAGAGGCATCCTCACCCT-3' } \\
\text { 5'-TACATGGCTGGGGTGTTGAA-3' }\end{array}$ & 216 \\
\hline Syncytin-2 & $\begin{array}{l}\text { 5'-CTCCTGGCCATCATGCTCTC-3' } \\
\text { 5'-GGCCACCAAGATGAGAAA-3' }\end{array}$ & 129 \\
\hline PR-B & $\begin{array}{l}\text { 5'-AGCAGTCCGCTGTCCTTTTCT-3' } \\
\text { 5'-CCTGAAGTTTCGGCCATACCT-3' }\end{array}$ & 196 \\
\hline PR-A/B & $\begin{array}{l}\text { 5'-TTTCGACCTCCAAGGACCAT-3' } \\
\text { 5'-AGCCCACAATACAGCTTCGAG-3' }\end{array}$ & 254 \\
\hline
\end{tabular}

MTT cell viability assay. The MTT solution (Sigma-Aldrich, Belgium) was added to the cells and incubated for $3 \mathrm{~h}$ and $30 \mathrm{~min}$ at $37^{\circ} \mathrm{C}$. The resulting MTT formazan crystals were dissolved using MTT solvent, an anhydrous isopropranol solution, and the absorbance was measured spectrophotometrically at 560 and $620 \mathrm{~nm}$. This was done in order to get the absolute absorbance value to plot the graphs. The experiments were performed in triplicate wells.

$P R-B$ staining using ImageStream analysis. BeWo cells were cultured to $80 \%$ confluency. Cells were then collected, and the pellet formed was resuspended in $1 \mathrm{ml}$ PBS followed by centrifugation for $5 \mathrm{~min}$ at $1,500 \mathrm{rpm}$. Ice-cold $50 \%$ methanol:50\% acetone solution $(1 \mathrm{ml})$ was added to the cells and incubated on ice for $10 \mathrm{~min}$. A centrifugation step for $5 \mathrm{~min}$ at 5,000 rpm followed in order to remove the fixative and a brief wash with PBS was performed. A further spin at $5,000 \mathrm{rpm}$ for $5 \mathrm{~min}$ was followed with removal of PBS. Cells were incubated with $1 \mathrm{ml}$ of $0.2 \%$ milk and $0.1 \%$ Tween-20 in PBS and incubated at room temperature for $1 \mathrm{~h}$ on a roller. A spin at $5000 \mathrm{rpm}$ for $5 \mathrm{~min}$ was performed to remove the block. The primary antibody PR-B (Santa Cruz Biotechnology, SC811, mouse: 1:100 dilution) was added and incubated for $1 \mathrm{~h}$ at room temperature. The primary antibody was removed by performing a spin at 5,000 rpm for $5 \mathrm{~min}$. Two washes in $0.1 \%$ Tween-20 PBS followed prior to incubating cells with an anti-mouse TRITC secondary antibody (1:150 dilution) for $1 \mathrm{~h}$ at room temperature. The secondary antibody was removed, cells were rinsed once with $0.1 \%$ Tween-20 PBS prior to adding the counterstain.
Progesterone-BSA conjugated FITC staining using ImageStream analysis. BeWo cells were cultured to $80 \%$ confluency, cells were collected, and the pellet formed was resuspended in $1 \mathrm{ml} \mathrm{Ham} \mathrm{F12} \mathrm{media.} \mathrm{Cells} \mathrm{were} \mathrm{then} \mathrm{transferred} \mathrm{to} \mathrm{a} 1.5 \mathrm{ml}$ Eppendorf tube. P4 conjugated to BSA-FITC was added at $100 \mathrm{nM}$ concentration to the cells and incubated at $37^{\circ} \mathrm{C}$ for $5 \mathrm{~min}$ followed by centrifugation for $5 \mathrm{~min}$ at $1,500 \mathrm{rpm}$. The cells were then briefly washed with $0.1 \%$ Tween-20, PBS and centrifuged for $5 \mathrm{~min}$ at $1,500 \mathrm{rpm}$. Paraformaldehyde $4 \%(0.5 \mathrm{ml})$ was added to the cells and incubated at room temperature for $10 \mathrm{~min}$. The cells were then centifuged at $5,000 \mathrm{rpm}$ for $5 \mathrm{~min}$, the fixative was carefully removed and the cells were briefly washed in PBS. The cells were centrifuged again on order to remove the PBS, fresh PBS was added followed by the addition of the counterstain.

\section{Results}

Syncytialization of BeWo cells. BeWo cells are fusigenic and when they are fully syncytialized they resemble a third trimester placental model; whereas, undifferentiated BeWo cells are similar in morphology to primary trophoblast cultures. In our experimental model, syncytialization was achieved by incubation with forskolin up to $72 \mathrm{~h}$. The syncytialization model on BeWo cells was evaluated in detail, prior to providing a comprehensive expression map of PRs in the syncytialized (syncytiotrophoblasts) and non-syncytialized BeWo cells. We have recently provided a detailed evaluation of the syncytialization model of BeWo cells using different approaches at both the gene and protein levels. Briefly, PCNA 
A.

$\begin{array}{lllll}1 & 2 & 3 & 4 & 5\end{array}$

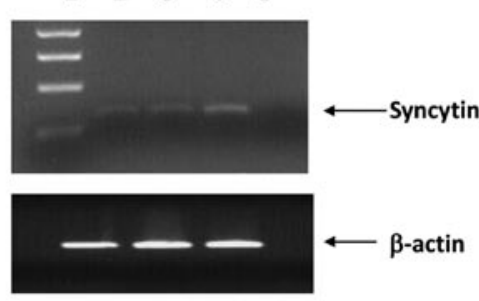

C.

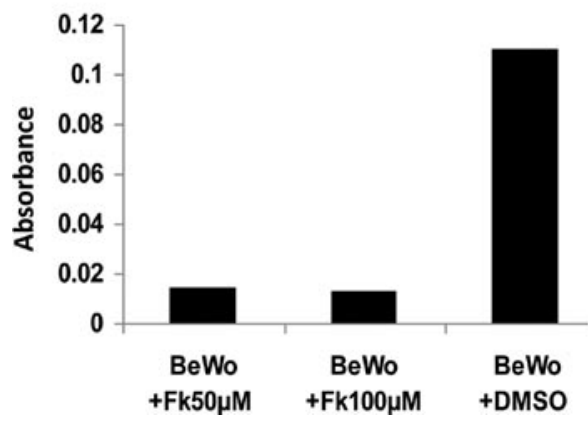

B.

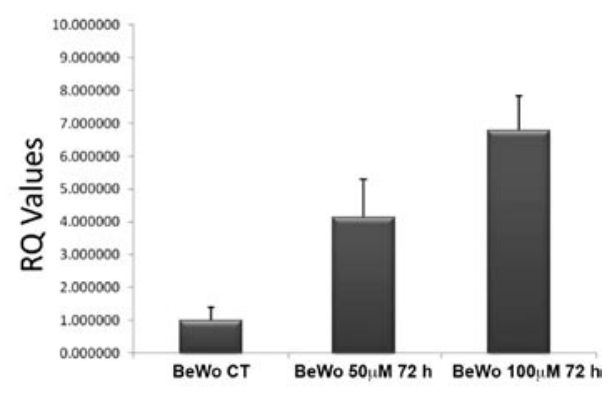

D.

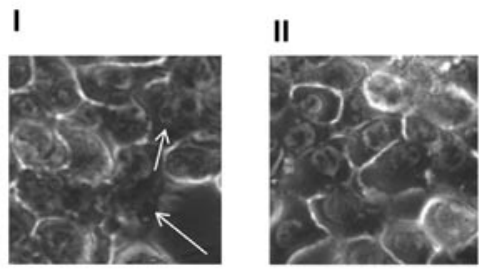

Figure 1. (A) Gene expression of syncytin corrected over $\beta$-actin. Lane 1, DNA ladder; lane 2, cDNA from human placenta; lane 3, cDNA from non- syncytialized (untreated) BeWo cells; lane 4, from syncytialized (forskolin treated; $50 \mu \mathrm{M}$ for $72 \mathrm{~h}$ ) BeWo cells; and lane 5, negative control. (B) Effect of syncytialization on the expression of syncytin assessed by Q-PCR in treated BeWo (forskolin $50 \mu \mathrm{M}$ for $72 \mathrm{~h}$ ) against control BeWo (BeWo CT). (C) The MTT assay revealed that treatment of BeWo cells with forskolin inhibited cell growth and proliferation. (D) Microscopy revealed decreases in the overall content of the cell membrane in forskolin-treated BeWo cells (I) when compared with the untreated control (BeWo + DMSO; II). All experiments were performed in triplicate. White arrows indicate the fusion of the cell membranes.

A.

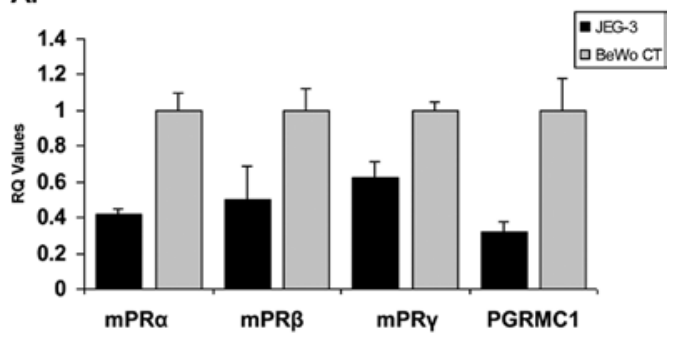

B.

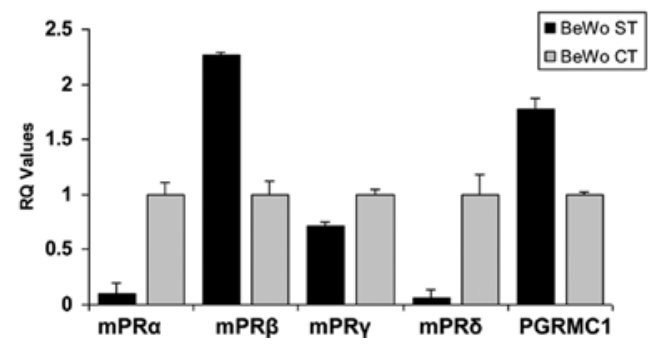

Figure 2. (A) Q-PCR for $m P R \alpha, m P R \beta, m P R ~ \gamma$ and PGRMC1 in JEG-3 vs. control untreated BeWo cells (BeWo CT). (B) Effect of syncytialization on the expression of mPRs and PGRMC1 assessed by Q-PCR in treated BeWo (BeWo ST, forskolin $50 \mu \mathrm{M}$ for $72 \mathrm{~h}$ ) against control BeWo cells (BeWo CT). All experiments were performed in triplicate.

(proliferating cell nuclear antigen) was down-regulated in the treated cells when compared to the non-treated cells both at the mRNA and protein level. Evaluation of the cell membrane marker cadherin, was also performed to determine whether there was a decrease of the total plasma membrane in response to forskolin treatment. Subsequent Western blotting demonstrated down-regulation of cadherin protein levels in BeWo cells treated with forskolin (24).

For this study, we used syncytin gene expression as an additional marker for syncytialization. BeWo cells treated with forskolin $(50$ or $100 \mu \mathrm{M})$ induced the expression of syncytin, a marker of syncytialization (Fig. 1A and B). An MTT assay was also carried out in BeWo cell cultures 48 and $72 \mathrm{~h}$ after initial plating to assess cell viability after exposing the cells to forskolin treatment. The principle of the MTT test is based on the ability of viable cells to produce formazan from the active cleavage of the tetrazolium salt by the functional mitochondria. The MTT assay revealed that treatment of BeWo cells with forskolin (50 or $100 \mu \mathrm{M})$ for $48 \mathrm{~h}$ significantly inhibited cell growth (Fig. 1C). Similar data were evident at $72 \mathrm{~h}$ (data not shown). This was further corroborated by the appearance of BeWo cells using an inverted microscope, by which loss of the plasma membrane was evident in forskolin-treated cells, as they became amorphous and multinucleated (Fig. 1D).

Effect of syncytialization on of mPRs, PGRMC1 and PR gene expression. For this study, fully syncytialized $(50 \mu \mathrm{M}$ forskolin for $72 \mathrm{~h}$ ) and non-syncytialized (untreated) BeWo cells were used, as well as cDNA from JEG-3 cells. Prior to measuring gene differences during syncytialization, the quantitative RT-PCR conditions were assessed. All genes were amplified at similar rates, giving rise to a single PCR product. This was evident from a single peak during the melting curve 
A.

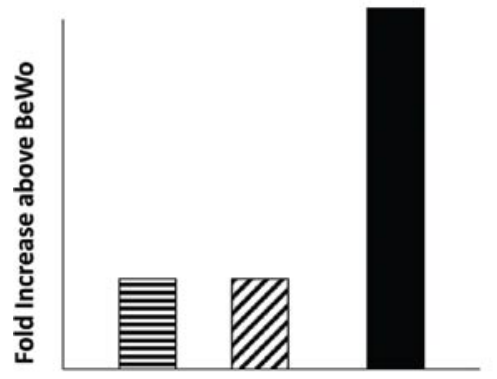

BeWo CT BeWo ST JEG-3
B.

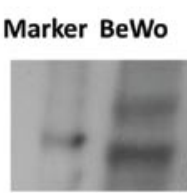

Marker JEG-3

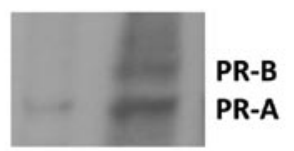

C.
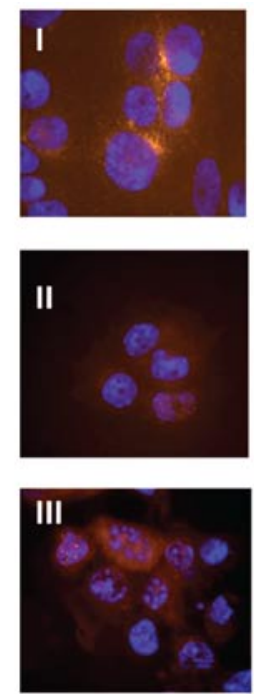

1
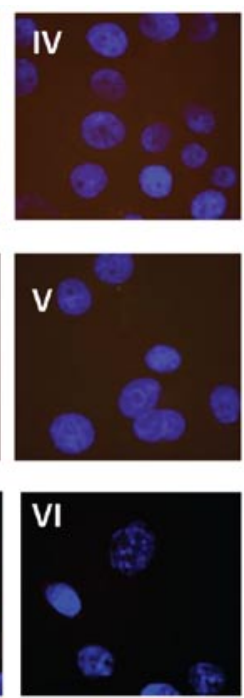

E.

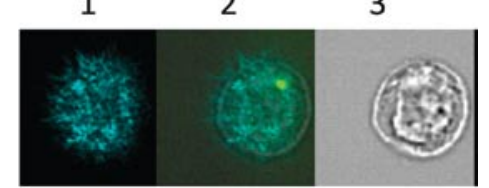

Figure 3. (A) Semi-quantitative RT-PCR for PR-B corrected over $\beta$-actin, demonstrated that PR-B gene expression is not altered in forskolin-treated or untreated BeWo cells. (B) Western blotting of the expression of PR-A and PR-B in cell lysates from BeWo and JEG-3 cells. Total cell lysates were resolved on a gradient (4-20\%) polyacrylamide gel. (C) Immunofluorescence analysis for PR-B in JEG-3 (I), non-syncytialized BeWo (II) and syncytialized BeWo cells (III). Images in (IV), (V) and (VI) are negative controls of (I), (II) and (III). (D) ImageStream analysis of PR-B in two representative BeWo cells. D1 is scatter cell imaging, D2 is the brightfield image of the cells, and D3 is the specific staining (red) for PR-A/B (blue arrows). (E) Binding of FITC-P4-BSA on the cell membrane of BeWo cells. E1 scatter cell imaging, E2 shows FITC-P4-BSA staining (white arrow; cell membrane), and E3 is the brightfield image of the cell.

analysis as well as a single band when the PCR products were resolved on agarose gels (data not shown).

Quantitative RT-PCR revealed that there was a significant down-regulation of $\mathrm{mPR} \alpha(90 \%), \mathrm{mPR} \delta$ in $(99 \%)$ and a more modest decrease (28\%) for mPR $\gamma$ in forskolin-treated BeWo (BeWo ST) cells when compared with non-syncytialized BeWo (BeWo CT) cells (Fig. 2B). Interestingly, forskolin pre-treatment led to a significant up-regulation of $\mathrm{mPR} \beta$ (127\%) and PGRMC1 (77\%) in syncytialized BeWo cells (Fig. 2B). When non-differentiated BeWo cells (fusigenic) were compared to JEG-3 (non-fusigenic), there was a significantly lower expression of $\operatorname{mPR} \alpha(58 \%), \operatorname{mPR} \beta(50 \%), \mathrm{mPR} \gamma$ (38\%), PGRMC1 (68\%) (Fig. 2A) in JEG-3 cells, whereas there was no expression of mPR $\delta$ in JEG-3 cells (data not shown). With regards to $\mathrm{mPR} \varepsilon$, no apparent expression of this gene was detected in all cDNAs tested (data not shown).

Semi-quantitative analysis was then used to assess the expression of nuclear PR-B. This approach was chosen due to the presence of other PR splice variants (e.g. PR-A). RT-PCR analysis followed by correction over $\beta$-actin revealed that the expression of PR-B did not varry between non-syncytialized and syncytialized BeWo cells (Fig. 3A). Interestingly, in JEG-3 there was a 4-fold increase in the expression of PR-B when compared to BeWo (Fig. 3A).

We then used a specific PR-B antibody and immunofluorescence analysis to assess the localisation of the nuclear receptor in our preparations. In JEG-3 cells, PR-B was localised primarily in the cytoplasm (perinuclear staining) with some nuclear localisation (Fig. 3C, panel I), whereas in non-syncytialized and syncytialized BeWo cells, PR-B was localised primarily in the nucleus (Fig. 3C, panels II and III). Western blotting was then utilised to further investigate the expression of PR isoforms in these cell lines. Specific bands $\sim 116$ and $94 \mathrm{kDa}$ were detected in all preparations, indicative of the expression of the full-length PR-B and the truncated PR-A, respectively (Fig. 3B). The specificity of Western blotting was further verified by using a PR-B specific antibody that produced a single immunoreactive band at $116 \mathrm{kDa}$ (data not shown).

ImageStream analysis. Following the immunofluorescence analysis, we used the state-of-the-art ImageStream technology that combines flow cytometry with cell imaging for parallel quantification and visualization of single cells. Strong nuclear expression of PR was evident in most of 4,448 single cells studied (Fig. 3D). In addition, incubation of cells with fluorescently conjugated $\mathrm{P} 4$ bound to the impermeable moiety BSA resulted in binding on the cell membrane of BeWo cells, indicative of membrane progesterone binding sites (Fig. 3E).

Cellular localisation of mPRs, PGRMC1 and PRs in JEG-3 and BeWo cells. The protein expression and cellular localisation of mPRs, and PGRMC1 was also assessed in JEG-3, non-syncytialized and syncytialized BeWo cells, using immunofluorescence analysis. In JEG-3 cells, mPR $\alpha$ was expressed in both the plasma membrane and cytoplasm, whereas mPR $\beta$ 


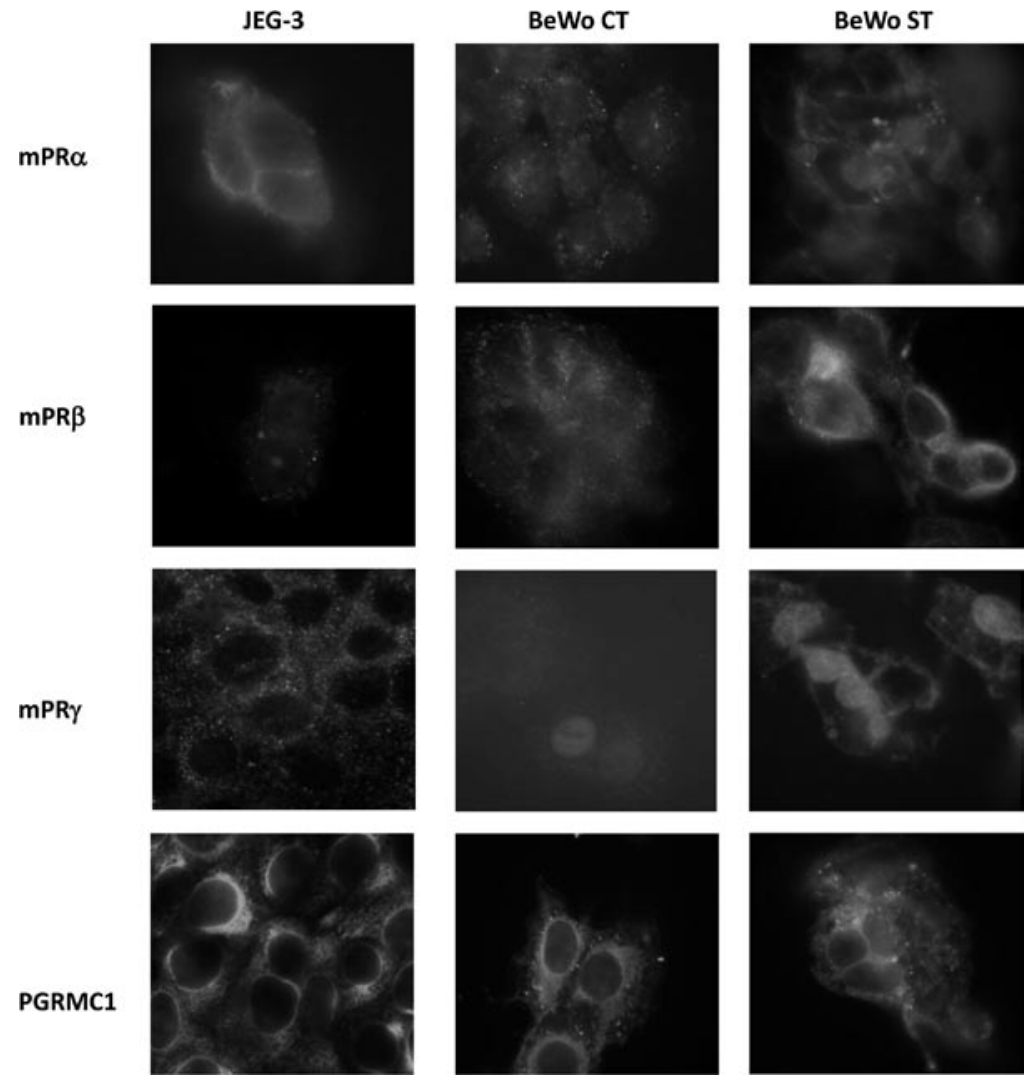

Figure 4. Immunofluorescence analysis for $\mathrm{mPR} \alpha, \mathrm{mPR} \beta, \mathrm{mPR} \gamma$, and PGRMC1 in JEG-3 cells, non-syncytialized (BeWo CT) and syncytialized cells (BeWo ST).

and $\mathrm{mPR} \gamma$ showed a predominantly cytoplasmic localisation, PGRMC1 a strong perinuclear staining and PR-B a primarily cytoplasmic localisation (perinuclear staining) with some nuclear localisation (Fig. 4). In non-syncytialized (undifferentiated) BeWo cells, mPR $\alpha, \operatorname{mPR} \beta, \operatorname{mPR} \gamma$ and PGRMC1 were localised in the plasma membrane as well as in the cytoplasm (Fig. 4). When BeWo cells were treated with forskolin for $72 \mathrm{~h}$, there was an evident decrease of plasma membrane followed by formation of polynucleated syncytia. In these cells, $\mathrm{mPR} \alpha$ is predominantly localised in the cytoplasm (Fig. 4).

We further assessed the localisation of mPRs in clinical samples. Immunofluorescence analysis of mPRs in human placental sections revealed a differential cellular localisation and distribution. mPR $\alpha$ was localised around syncytiotrophoblasts with a cytoplasmic localisation, whereas $\operatorname{mPR} \beta$ was expressed both in the cytoplasm as well as on the plasma membrane (also around syncytiotrophoblasts) (Fig. 5). Interestingly, $\mathrm{mPR} \gamma$ was expressed in both syncytiotrophoblasts and cytotrophoblasts. The expression was stronger around syncytiotrophoblasts, where $\operatorname{mPR} \gamma$ appeared to be expressed mainly on the plasma membrane (Fig. 5).

\section{Discussion}

In this study we provide a detailed map of the PR expression at the gene and protein levels in two human choriocarcinoma cell lines with different fusigenic capacities. Moreover, we assessed the effect of forskolin as a syncytialization agent in the expression of these genes.
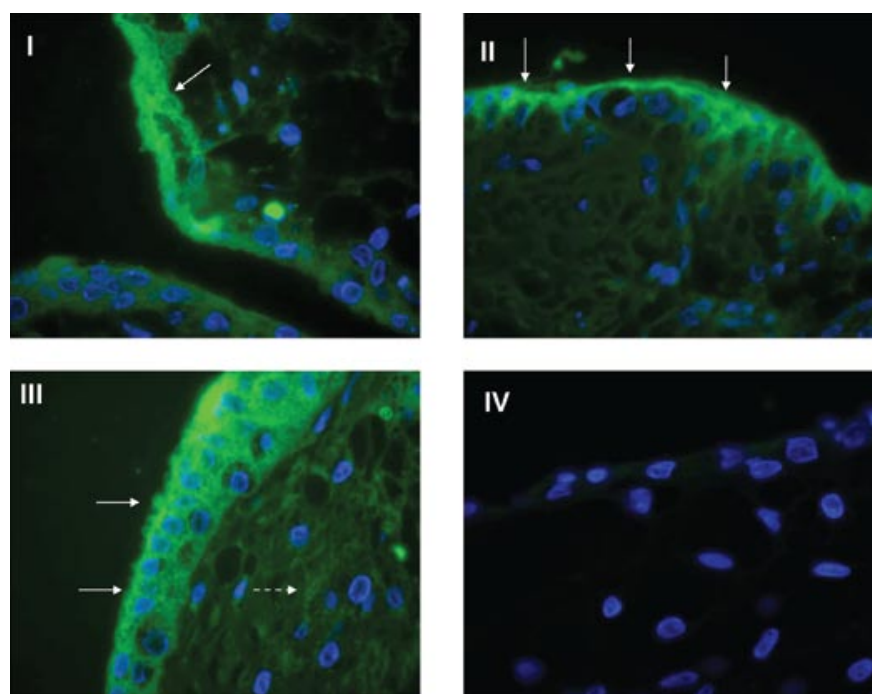

Figure 5. Immunofluorescence analysis revealed strong cytoplasmic localization for $\mathrm{mPR} \alpha$ (Panel I), whereas mPR $\beta$ (Panel II), and mPR $\gamma$ (Panel III) are distributed in the membrane (white arrows) and cytoplasm of human placental tissue sections. mPR $\gamma$ is also expressed in cytotrophoblasts (dotted arrow). Panel IV is the negative control.

Quantitative RT-PCR analysis revealed that in syncytialized BeWo cells, $\mathrm{mPR} \alpha, \mathrm{mPR} \delta$ and $\mathrm{mPR} \gamma$ were down-regulated, whereas $\mathrm{mPR} \beta$ and PGRMC1 were up-regulated when compared to non-syncytialized BeWo cells. The data indicate that mPRs and PGRMC1 may be implicated in the developmental events leading to placental syncytialization. These 
results corroborate a recent study showing that forskolin altered the expression of several genes during syncytialization of BeWo cells. Using differential display RT-PCR analysis and Northern blot analysis of genes such as SLPI, ELF-1 $\alpha-1$ and prolyl 4-hydroxylase $\beta$ were up-regulated with forskolin treatment (25).

When PR gene expression was compared between nonsyncytialized BeWo and JEG-3 cells, $\mathrm{mPR} \alpha, \mathrm{mPR} \beta, \mathrm{mPR} \gamma$, and PGRMC1 mRNa levels were significantly lower in JEG-3 cells. No expression of $\mathrm{mPR} \varepsilon$ was detected using quantitative or standard RT-PCR analyses even after 40 cycles of amplification. Further studies in a wider tissue repertoire will provide better insight into the expression patterns of these genes.

The JEG-3 cell line does not have a fusigenic capacity and responds poorly to inducers of syncytialization. Phenotypically JEG-3 cells resemble the undifferentiated cytotrophoblasts, making it a suitable in vitro model to investigate first trimester placental function (26). As an additional control, the effect of forskolin (50 $\mu \mathrm{M}$ or $100 \mu \mathrm{M}$, up to $72 \mathrm{~h}$ ) was tested on JEG-3 cells. Treatment with forskolin did not induce the expression of the syncytin gene (unpublished data). These data therefore verify that this cell line does not fuse under stimulation of cAMP. Although both the JEG-3 and BeWo cell lines may represent a cytotrophoblastic state, there is evidence that they have inherent gene differences. Microarray analysis revealed that many transcripts were differentially expressed between BeWo and JEG-3 cells (27), suggesting that each cell line may vary in its capacity to respond to steroid hormones.

As mentioned, the human placenta expresses many PR isoforms (15). In our study, we provide evidence that JEG-3 cells express the full-length PR-B as demonstrated by RT-PCR and Western blot analyses. Moreover, our data corroborate a recent study that both the PR-A and PR-B proteins are expressed in BeWo cells (28). The presence of multiple PR isoforms may be indicative of the diversity in progesterone responses. For example, in BeWo cells transfected with either PR-B or PR-A, these isoforms were found to differentially regulate the expression of the breast cancer resistance protein (29).

Immunofluorescence analysis revealed strong cytoplasmic staining for $\mathrm{mPR} \alpha$ in both cell lines. In the human placenta mPR $\alpha$ was localised almost exclusively around syncytiotrophoblasts. This is in agreement with a study by Fernandes et al, demonstrating that $\mathrm{mPR} \alpha$ expression is restricted to the syncytiotrophoblast at the periphery of term chorionic villi (9). mPR $\beta$ had a similar immunoreactivity pattern, whereas $\mathrm{mPR} \gamma$ was primarily expressed on the plasma membrane of syncytiotrophoblasts. With regards to PGRMC1, it has been previously shown that it is expressed in smooth muscle cells of the placental vasculature, villous capillaries and the syncytiotrophoblast (16). Interestingly, a different pattern of cellular localisation of mPRs and PGRMC1 was detected upon treatment with forskolin. For example, in non-syncytialized BeWo cells, $\mathrm{mPR} \alpha, \operatorname{mPR} \beta, \mathrm{mPR} \gamma$ and PGRMC1 were observed to have a membranous and cytoplasmic localisation, whereas in syncytialized cells mPR $\alpha$ was predominantly localised in the cytoplasm. This could be due to the changes that occur at plasma membrane conent following forskolin treatment.
Since receptor phosphorylation can alter its expression, translocation, or function (30), we then studied in silico the potential of cyclic AMP-dependent protein kinase A (PKA) to directly phosphorylate the mPRs and PGRMC1 and as a result alter their cellular localisation. For this phosphospecific analysis the pkaPS prediction tool (http://mendel.imp. univie.ac.at/sat/pkaPS) was utilised to detect potential serine threonine $(\mathrm{S} / \mathrm{T})$ residues. The scoring function of the pkaPS predictor can confidently discriminate PKA phosphorylation sites from $\mathrm{S} / \mathrm{T}$ with non-permissive sequence environments (sensitivity of $\sim 96 \%$ at a specificity of $\sim 94 \%$ ) (31). No PKA phosphorylation sites were predicted for $\operatorname{mPR} \alpha, \operatorname{mPR} \beta$, mPR $\gamma$ or PGRMC1, thus suggesting that activation of PKA by forskolin in BeWo cells cannot induce any direct posttranslational modifications of these receptors. Pretreatment of BeWo with specific PKA or adenylyl cyclase (AC) inhibitors will provide a better understanding of the extent of the involvement of the AC/cAMP/PKA pathway in the cellular localisation of these receptors.

With regards to PR-B, in JEG-3 and non-syncytialized BeWo cells it appeared to have a cytoplasmic and nuclear localisation, whereas in forskolin-treated BeWo the localisation was primarily nuclear. This is in agreement with data indicating that PR-B distributes between the cytoplasm and the nucleus (32). Interestingly, elevation of cAMP can activate certain steroid receptors and augment the ligand-dependent activation. Rowan et al, have shown that 8-bromo-cyclic AMP induces phosphorylation of two sites in SRC-1 that facilitate ligand-independent activation of the chicken PR and are critical for the functional cooperation between SRC-1 and the CREB binding protein (33). Future studies should determine whether different inducers of syncytialization in BeWo cells can affect the activity of PRs.

To date, a few studies have provided evidence of progesterone-mediated actions in human choriocarcinoma cell lines. For example, progesterone inhibited folic acid transport (34) and down-regulated human organic anion transporter 4 (hOAT4) transport activity in human placental BeWo cells (35). However, none of these studies clearly identifies the receptor mechanism via which these progesterone effects are mediated. Our data provide evidence for the expression of multiple PRs in human placental cell lines, suggestive of a higher order of complexity of progesterone-mediated responses. However, we were unable to study further protein expression and cellular distribution of $\mathrm{mPR} \delta$ and $\mathrm{mPR} \varepsilon$, due to lack of commerciallyavailable antibodies. While this manuscript was in preparation, a study using transmission electron microscopy demonstrated that PRs can be present on the membrane as well as in the nucleus of human spermatozoa (36). Therefore, future work should concentrate on further dissecting the involvement of nuclear receptors in non-genomic responses and determine the cellular localisation of these receptors in human placentas from complicated pregnancies such as pre-eclampsia, intrauterine growth retardation or gestational diabetes.

\section{Acknowledgements}

This research was funded by the National Institutes of Health (Grant ESO12961) to P.T. and E.K., and by The Society for Endocrinology to E.Z. 


\section{References}

1. Niswender GD, Juengel JL, Silva PJ, Rollyson MK and McIntush EW: Mechanisms controlling the function and life span of the corpus luteum. Physiol Rev 80: 1-29, 2000.

2. Bromley T: Non-genomic progesterone receptors in the mammalian ovary: some unresolved issues. Reproduction 125: $3-15,2003$.

3. Larsen WJ: Fetal development and the fetus as patient. In: Human Embryology. Sherman LS, Potter SS and Scott WJ (eds). 3rd edition, Churchill Livingstone, pp481-498, 2001.

4. Hirata S, Shoda T, Kato J and Hoshi K: Isoform/variant mRNAs for sex steroid hormone receptors in humans. Trends Endocrinol Metab 14: 124-129, 2003.

5. Falkenstein E, Tillmann HC, Christ M, Feuring $M$ and Wehling M: Multiple actions of steroid hormones-A focus on rapid, nongenomic effects. Pharmacol Rev 5: 513-556, 2000.

6. Watson CS and Gametchu B: Proteins of multiple classes may participate in nongenomic steroid actions. Exp Biol Med 228: 1272-1281, 2003.

7. Thomas P: Characteristics of membrane progestin receptor alpha (mPR alpha) and progesterone membrane receptor component 1 (PGRMC1) and their roles in mediating rapid progestin actions. Front Neuroendocrinol 29: 292-312, 2008.

8. Zhu Y, Rice CD, Pang Y, Pace M and Thomas P: Cloning, expression, and characterisation of a membrane progestin receptor and evidence it is an intermediary in meiotic maturation of fish oocyte. Proc Natl Acad Sci USA 100: 2231-2236, 2003.

9. Fernandes MS, Pierron V, Michalovich D, et al: Regulated expression of putative membrane progestin receptor homologues in human endometrium and gestational tissues. J Endocrinol 187: 89-101, 2005.

10. Smith JL, Kupchak BR, Garitaonandia I, et al: Heterologous expression of human mPR, mPR, mPR in yeast confirms their ability to function as membrane progesterone receptors. Steroids 73: $1160-1173,2008$

11. Peluso JJ, Pappalardo A, Losel R and Wehling M: Progesterone membrane receptor component 1 expression in the immature rat ovary and its role in mediating progesterone's antiapoptotic action. Endocrinology 147: 3133-3140, 2006.

12. Falkenstein E, Meyer C, Eisen C, Scriba PC and Wehling M: Full-length cDNA sequence of a progesterone membranebinding protein from porcine vascular smooth muscle cells. Biochem Biophys Res Commun 229: 86-89, 1996.

13. Peluso JJ: Multiplicity of progesterone's actions and receptors in the mammalian ovary. Biol Reprod 75: 2-8, 2006

14. Gard PR: The ovaries and the female reproductive system. In: Human Endocrinology. Wrigglesworth J (ed). Taylor \& Francis Ltd., London, pp147-170, 1998.

15. Taylor AH, McParland PC, Taylor DJ and Bell SC: The cytoplasmic $60 \mathrm{kDa}$ progesterone receptor isoform predominates in the human amniochorion and placenta at term. Reprod Biol Endocrinol 13: 7-22, 2009.

16. Zhang L, Kanda Y, Roberts DJ, et al: Expression of progesterone receptor membrane component 1 and its partner serpine 1 mRNA binding protein in uterine and placental tissues of the mouse and human. Mol Cell Endocrinol 287: 81-89, 2008.

17. Loregger T, Pollheimer J and Knofler M: Regulatory transcription factors controlling function and differentiation of human trophoblast - a review. Placenta 17: 104-110, 2003.

18. Huang FD, Kung FL, Tseng YC, et al: Regulation of protein expression and function of OCTN2 in forskolin-induced syncytialization in BeWo cells. Placenta 30: 187-194, 2009.

19. Knipp GT, Audus KL and Soares MJ: Nutrient transport across the placenta. Adv Drug Deliv Rev 38: 41-58, 1999.

20. Enders AC and Blankenship TN: Comparative placental structure. Adv Drug Deliv Rev 38: 3-15, 1999.
21. Jansson T and Powell TL: Role of the placenta in fetal programming: underlying mechanisms and potential interventional approaches. Clin Sci 113: 1-13, 2007.

22. Al-Nasiry S, Spitz B, Hanssens M, Luyten C and Pijnenborg R: Differential effects of inducers of syncytialization and apoptosis on BeWo and JEG-3 choriocarcinoma cells. Hum Reprod 21: 193-201, 2006.

23. Karteris E, Chen $\mathbf{J}$ and Randeva HS: Expression of human prepro-orexin and signaling characteristics of orexin receptors in the male reproductive system. J Clin Endocrinol Metab 89: 1957-1962, 2004

24. Mparmpakas D, Zachariades E, Foster H, Kara A, Harvey A, Goumenou A and Karteris E: Expression of mTOR and downstream signalling components in the JEG-3 and BeWo human placental choriocarcinoma cell lines. Int J Mol Med 25: 65-69, 2010.

25. Neelima PS and Rao AJ: Gene expression profiling during Forskolin induced differentiation of BeWo cells by differential display RT-PCR. Mol Cell Endocrinol 281: 37-46, 2008.

26. Ntrivalas E, Kwak-Kim J, Beaman K, Mantouvalos H and Gilman-Sachs A: An in vitro coculture model to study cytokine profiles of natural killer cells during maternal immune celltrophoblast interactions. J Soc Gynecol Investig 13: 196-202, 2006.

27. Burleigh DW, Kendziorski CM, Choi YJ, et al: Microarray analysis of BeWo and JEG3 trophoblast cell lines: identification of differentially expressed transcripts. Placenta 28: 383-389, 2007.

28. Yasuda S, Kobayashi M, Itagaki S, Hirano T and Iseki K: Response of the ABCG2 promoter in T47D cells and BeWo cells to sex hormone treatment. Mol Biol Rep 36: 1889-1896, 2009.

29. Wang H, Unadkat JD and Mao Q: Hormonal regulation of BCRP expression in human placental BeWo cells. Pharm Res 25: 444-452, 2008.

30. Pollock VV, Pastoor TE and Wecker L: Cyclic AMP-dependent protein kinase (PKA) phosphorylates Ser362 and 467 and protein kinase C phosphorylates Ser550 within the M3/M4 cytoplasmic domain of human nicotinic receptor alpha4 subunits. J Neurochem 103: 456-466, 2007.

31. Neuberger G, Schneider G and Eisenhaber F: pkaPS: prediction of protein kinase A phosphorylation sites with the simplified kinase-substrate binding model. Biol Direct 2: 1, 2007.

32. Ellmann S, Sticht H, Thiel F, Beckmann MW, Strick R and Strissel PL: Estrogen and progesterone receptors: from molecular structures to clinical targets. Cell Mol Life Sci 66: 2405-2426, 2009.

33. Rowan BG, Garrison N, Weigel NL and O'Malley BW: 8-Bromocyclic AMP induces phosphorylation of two sites in SRC-1 that facilitate ligand-independent activation of the chicken progesterone receptor and are critical for functional cooperation between SRC-1 and CREB binding protein. Mol Cell Biol 20: 8720-8730, 2000.

34. Keating E, Gonçalves P, Lemos C, et al: Progesterone inhibits folic acid transport in human trophoblasts. J Membr Biol 216: 143-152, 2007.

35. Zhou F, Xu W, Tanaka K and You G: Comparison of the interaction of human organic anion transporter hOAT4 with PDZ proteins between kidney cells and placental cells. Pharm Res 25: 475-480, 2008.

36. De Amicis F, Guido C, Perrotta I, Avena P, Panza S, Andò S and Aquila S: Conventional progesterone receptors (PR) B and PRA are expressed in human spermatozoa and may be involved in the pathophysiology of varicocoele: a role for progesterone in metabolism. Int J Androl: October 14, 2010 (Epub ahead of print). 